\title{
Habilidades socioemocionales, lectura y escritura en la transición de educación inicial a primer año escolar
}

\author{
Socio-emotional skills, reading and writing in the transition from \\ initial education to the first school year
}

\section{Tania Presa Rodríguez*}

\footnotetext{
* Maestra. Licenciada en Ciencias de la Educación (Facultad de Humanidades y Ciencias de la educación, UDELAR). Magíster en Psicología y Educación (Facultad de Psicología, UDELAR). Doctoranda en Psicología (Facultad de Psicología, UDELAR). Docente del Consejo de Formación en Educación en las carreras de Maestro de Primaria y de Primera Infancia. Investigadora en el campo de las habilidades socioemocionales.

$\triangle \cdot$ lic_taniapresa@hotmail.com https://orcid.org/0000-00020865-8478
}

RECIBIDO: 14.3 .2020

ACEPTADO: 19.4 .2020

\section{Resumen}

El trabajo reflexiona sobre la relación entre habilidades socioemocionales y aprendizaje de la lectura y la escritura en su etapa inicial. Dicha relación es discutida en la transición educativa de nivel inicial a primer año. El recorrido teórico se enfoca en las habilidades socioemocionales desde una perspectiva de valoración cognitiva, en el contexto actual del movimiento del affective turn, e identifica algunos aspectos clave para pensar el proceso de construcción de la emocionalidad en la historiografía educativa. El planteo toma como premisa la influencia positiva (facilitadora) o negativa (obstaculizadora) de lo emocional en las experiencias de enseñanza, aprendizaje y evaluación de las prácticas de lectura y escritura durante el primer año escolar. Para finalizar, se explora como estrategia educativa la implementación de programas de intervención en habilidades socioemocionales a partir de la reflexión de sus fundamentos.

Palabras clave: educación de la primera infancia, afectividad, desarrollo afectivo, lectura.

\section{Abstract}

The article reflects on the relationship between socioemotional skills and literacy acquisition during the educational transition from pre-school levels to first 
school form. The theoretical journey focuses on socio-emotional skills from a cognitive assessment perspective, in the current context of the Affective Turn movement, and identifies some key aspects to think about the process of construction of emotionality in educational historiography. The approach relies on the assumption of the positive (facilitating) or negative (impeding) influence of emotion on teaching, learning, and assessing literacy practices during the first school year. Finally, the implementation of socio-emotional skills programs of intervention is explored as an educational strategy, based on the reflection on its foundations.

Key words: early childhood education, affectivity, affective development, reading.

\section{Introducción}

La cultura de la escuela es un conjunto holístico de cogniciones y emociones en interacción.

(Escolano, A., Emociones y educación, 2018)

El presente artículo pone en discusión la relación entre habilidades socioemocionales y lenguaje, en las áreas de lectura y escritura. El análisis de dicha relación se enfoca en el campo de la educación inicial durante la transición de nivel cinco a primer año. En este marco se proponen dos interrogantes que guían el trabajo: ¿cómo influyen las habilidades socioemocionales en los desempeños esperados en lectura y escritura durante el primer año escolar? y ¿cómo promover una transición exitosa de inicial a primer año a través de experiencias de aprendizaje basadas en la estimulación de las habilidades socioemocionales?

Durante la primera infancia, las interacciones entre el niño y su entorno, así como las experiencias y los vínculos que de esas interacciones se promuevan, serán clave para su futuro desarrollo emocional y adquisición de aprendizajes. Contamos con evidencia que demuestra la influencia de las habilidades socioemocionales y su influencia en los desempeños académicos (Collaborative for Academic, Social, and Emotional Learning [CASEL], 2007; Durlak y Weissberg, 2011, Organización para la Cooperación y el Desarrollo Económicos [OCDE], 2019).

Estos aspectos vinculados a la importancia del trabajo sobre las habilidades socioemocionales desde la primera infancia, y en especial sobre el ciclo de educación inicial, requieren ser pensados en el proceso de transición educativa de nivel cinco a primer año. Allí, la transición aparece como un «periodo caracterizado por la interrelación de ansiedades, miedos, expectativas y tensiones, que necesitan ser elaboradas para que el 
niño pueda adaptarse a esa nueva situación» (Salinas-Quiroz, 2015, p. 3). La transición educativa ha de ser pensada desde una perspectiva de corresponsabilidad, en la que todos los actores involucrados formen parte activa del proceso. Es necesario adoptar políticas y mecanismos institucionales que consideren la transición educativa desde su multidimensionalidad. En esta línea, el Fondo de las Naciones Unidas para la Infancia (UNICEF, 2012) establece determinados principios a considerar al respecto:

- Continuidad de las propuestas y expectativas del nivel inicial al primer año.

- Involucramiento familiar y respeto por las prácticas y cuestiones culturales familiares.

- Participación de las familias en el aprendizaje temprano.

- Atención a las prácticas, actitudes, creencias y estimulación que el hogar pueda ofrecer.

Las políticas educativas actuales no contienen programas enfocados en la transición educativa de nivel inicial a primer año. Sí hay programas de transición en otras esferas de la educación pública; por ejemplo, en el Programa de Maestros Comunitarios (PMC), que lleva adelante una línea de trabajo referida a la transición educativa de sexto año de primaria a secundaria.

Cuando el alumno transita de inicial a primer año, ocurre una experiencia de quiebre, que se traduce tanto en los nuevos formatos y modalidad de trabajo a los que se enfrenta como en los logros académicos que se esperan de él. En este sentido, las expectativas de logros respecto a los aprendizajes en conocimientos clave, como el de la lectura y escritura, suelen ser motivos de repitencia durante el primer año escolar, por no encontrarse los niños con habilidades suficientes para afrontar dichos aprendizajes (Schiefelbein y Farrel, 1973; Kardonsky, Kovalsky y Segure, 1969; Bravo y Salas, 1976; Gazmuri, Milicic y Schmidt, 1978; Astaburruaga, Pereira y Schmidt citado por Montes Cruzat, Condemarín Grimberg y Rioseco Izquierdo, s/f).

De acuerdo a los resultados del Tercer Estudio Regional Comparativo y Explicativo (TERCE) aplicado por la UNESCO en 2014, de los escolares uruguayos de tercero y sexto año evaluados, uno de cada cuatro no cuenta con los conocimientos básicos deseados en lectura. Estos resultados motivaron al Consejo de Educación Inicial y Primaria a desarrollar políticas tendientes a intervenir tempranamente. Una de ellas la aplicación de un instrumento de evaluación del desarrollo infantil (INDI) que permite detectar durante el ciclo de educación inicial, en diferentes áreas (cognitiva, motora, socioemocional y de disposición para el aprendizaje), desempeños descendidos y dificultades tempranas, además de fortalezas (para ampliar véase Vásquez, 2019).

Las políticas de gestión y los principios rectores del CEIP apuntan a potenciar la educación inicial y proteger las trayectorias escolares (Terigi, 2009) mediante el seguimiento de los alumnos de primer año en el marco del denominado Proyecto de Primer 
Ciclo (de nivel inicial a tercer año), desplegando determinadas líneas de intervención tendientes a favorecer el desarrollo infantil temprano.

\section{Emociones y habilidades socioemocionales}

La emoción, como concepto complejo, refiere al proceso en el que interviene un estímulo desencadenante, externo o interno, y una consecución de cambios de orden subjetivo (la vivencia de la emoción), cognitivo (la valoración que el sujeto hace sobre la emoción), fisiológico (cambios producidos en el sistema nervioso central), expresivo (comunicación y exteriorización de las emociones) y la adaptación al entorno como aspecto motivacional involucrado (Elices, 2015).

Esta concepción de la emoción, más ligada a la dimensión psicobiológica, hemos de analizarla en virtud de los factores socioculturales. Así, toda emoción trae consigo «modos de expresión en los que se encarnan las culturas, en el pasado y en nuestra época» (Escolano, 2018, p. 26). Este aspecto no es menor, ya que en el último tiempo se viene percibiendo una especie de insistencia en la integración de lo emocional en el escenario escolar. Es a este movimiento que Escolano (2018) alude con el término affective turn, para referirse, justamente, a la iniciativa dada por educadores, investigadores y académicos de ponderar lo afectivo como aspecto fundamental de la formación de los sujetos. Autores como Noah W. Sobe (citado en Escolano, 2018) subrayan la necesidad de reflexionar sobre lo emocional desde una historiografía educativa capaz de hacer posible la construcción discursiva de una episteme consolidada desde la cual poder comenzar a fundamentar la educación emocional.

El impulso que a partir del siglo XXI ha tomado el lugar de lo emocional en el campo educativo es notorio, como también lo han sido las múltiples investigaciones y estudios que evidencian la idea de que los aspectos socioemocionales son imprescindibles para el buen desempeño de la trayectoria escolar de los sujetos (Durlak y Weissberg, 2011; Weissberg, Durlak, Domitrovich y Gullotta, 2015; Cotrufo, 2016). Además, el fortalecimiento de la dimensión emocional favorecería el logro de aprendizajes significativos, a la vez que un mejor manejo del sujeto en distintas situaciones a lo largo de toda su vida. Esta línea de estudio ha puesto de manifiesto el interés en dos dimensiones de relevancia: por un lado, a partir de los estudios de Mayer y Salovey (1997) sobre la influencia de la llamada inteligencia emocional sobre el rendimiento escolar y, por otro lado, la incorporación de la educación emocional en los programas curriculares (Fernández-Berrocal, Pérez, Repetto y Extremera, 2004; Bisquerra, 2008). En este sentido se puede considerar que aquellos sujetos emocionalmente competentes promoverán 
una mejor relación con los demás y ejercerán una ciudadanía responsable (Collaborative for Academic, Social, and Emotional Learning [CASEL], 2013; Elias, Parker, Kash, Weissberg y O’Brien, 2008).

En lo que respecta a las habilidades socioemocionales, revisiones actuales (Instituto Nacional de Evaluación Educativa [INEEd], 2017; 2018) demuestran la conjunción de distintas taxonomías para definirlas, lo que devela la dificultad para consensuar una terminología. El Instituto de Evaluación Educativa (INEEd) propone la siguiente definición:

Las habilidades socioemocionales son entendidas como una serie de herramientas cognitivas, emocionales y sociales que tienen como función la adaptación del individuo al entorno y que facilitan el desarrollo personal, el relacionamiento social, el aprendizaje y el bienestar. Se van configurando de forma dinámica a partir de la interacción del individuo con el contexto, a través de experiencias y aprendizajes, y en ese sentido son consideradas como el resultado de procesos de desarrollo, que se extienden a través de todo el ciclo vital. (INEEd, 2018, p. 18)

Esta perspectiva de corte amplio considera a las habilidades socioemocionales como evolutivas, dinámicas y capaces de ser modificadas a través de intervenciones específicas, a la vez que contempla su influencia directa en el logro de aprendizajes, el desarrollo del sujeto y el bienestar social.

\section{Emoción y valoración cognitiva}

Existen diferentes modelos explicativos de las emociones. En este trabajo se opta por el modelo cognitivo, que, a diferencia del conductista, pone sobre el escenario un aspecto que trasciende las conductas observables: la valoración. Ello implicaría el siguiente proceso a experimentar por el sujeto: se activa un determinado estímulo (de naturaleza externa o interna), el sujeto lo valora o evalúa y, como consecuencia, se produce la emoción. El mismo estímulo puede desencadenar diferentes emociones, dadas la dimensión subjetiva del proceso y la intervención de otros factores, ya sean ambientales, biológicos o vinculados a determinadas experiencias de aprendizaje (Elices, 2015). Ello explica que sujetos que experimentan un mismo estímulo hagan de él valoraciones diferentes y, como consecuencia, sus emociones puedan diferir.

En este enfoque se reconocen los aportes de autores fundantes del cognitivismo, quienes de alguna manera iniciaron la perspectiva de estudio a la que adherimos. Se puede considerar a Williams James (1945) como el primer referente de la perspectiva 
cognitiva respecto al estudio de las emociones, quien puso el foco en la relación entre las experiencias emocionales y la activación fisiológica del individuo.

Dentro de la psicología cognitiva, el modelo más reconocido es el promovido por Lazarus y Folkman (1984). Se reconoce también la relevancia de otros modelos cognitivos, como el modelo bifactorial de la emoción, basado en los postulados de Marañón (1985) y Schachter y Singer (1962), el cual relaciona el componente fisiológico con el cognitivo como indispensables para que se produzca la emoción.

En la primera versión de su teoría, Lazarus y Folkman (1984) propusieron el supuesto de que el sujeto transita por una valoración primaria que está enfocada en la emoción y una valoración secundaria que implica el afrontamiento de la situación y el despliegue de estrategias adaptativas. Más adelante, sobre la década del noventa, desarrollaron la teoría cognitiva-motivacional-relacional. En esta reformulación, la valoración primaria y la secundaria adoptan diferentes componentes. La valoración primaria implica que el sujeto se cuestione sobre la relevancia motivacional, es decir, sobre la pertinencia de la situación o experiencia en función de sus objetivos o metas. Otro aspecto que integra esta valoración primaria refiere a la congruencia motivacional, entendida no solo como la identificación de la relevancia de lo que se experimenta, sino en términos de funcionalidad para el logro de un determinado objetivo. Este tipo de valoración se podría sintetizar mediante dos interrogantes: ¿es importante esta situación dados mis objetivos?, ¿me ayuda a lograrlos?

La valoración secundaria implica los siguientes elementos: responsabilidad, estrategias de afrontamiento dirigidas al problema, estrategias de afrontamiento dirigidas a la emoción y expectativas de futuro. Podrían sintetizarse mediante las siguientes preguntas: ¿de quién es la culpa de lo que está sucediendo?, ¿cuento con los recursos para afrontar el problema?, ¿puedo manejar emocionalmente esta situación? y ¿qué posibilidades tengo de que la situación se modifique? (Elices, 2015).

Desde este enfoque cognitivo, toda emoción se encuentra vinculada a los procesos de valoración que el sujeto hace sobre ella. En dicha valoración intervienen factores vinculados a la personalidad del sujeto, a los del contexto en el que el sujeto se encuentra, así como a las experiencias de aprendizaje de las que participe.

\section{La perspectiva en torno a la lectura y la escritura}

Concebimos las prácticas de lectura y escritura desde una perspectiva amplia, como prácticas culturales, sociales y cognitivas. Perspectiva amplia significa aquí poner atención en los factores que influyen en su adquisición, tanto los cognitivos como los emocionales (Caballero y García-Lago, 2010). En este sentido: 
La lectura y la escritura son comportamientos inteligentes que le permiten al sujeto la realización de operaciones mentales complejas, estrechamente relacionadas con los distintos factores que integran la situación comunicativa concreta en la que se desarrollan. Así, la lectura y la escritura involucran las funciones psicológicas superiores: memoria, atención, gnosias y praxias. (Programa de Lectura y Escritura en Español [ProLEE], 2016, p. 9)

Tomando los aportes de ProLEE (Programa de Lectura y Escritura en Español de la ANEP), se explicitará a continuación la definición de lectura y escritura a la que se adhiere y los componentes que interactúan en dichos procesos. La lectura se entiende como:

Una construcción social y cultural que tiene lugar en un espacio intersubjetivo en el que los lectores manifiestan comportamientos, actitudes y trasfondos culturales respecto del acto de leer. Consideramos, además, que la lectura es una actividad inteligente que supone la interacción simultánea de las funciones psicológicas superiores, los diferentes niveles lingüísticos, el conocimiento del mundo, aspectos discursivos y metacognitivos. (ProLEE, 2016, p. 13)

A partir de esta definición se proponen como componentes de la lectura los siguientes: comportamiento lector, conocimiento del sistema de escritura, comprensión textual, conocimiento lingüístico y conocimiento discursivo. A la hora de ser evaluados, estos componentes sufrirán variaciones según el tipo de lector al que refieran. Como tipos de lectores es posible identificar a aquel que no ha ingresado al sistema de educación formal, al lector de nivel inicial que comienza la educación formal, al lector que transita por los primeros años de educación primaria, al que transita por los últimos años de primaria, al que finaliza el primer ciclo de educación media, al que finaliza el segundo ciclo de educación media y al que finaliza la educación terciaria (ProLEE, 2016). Por el objeto de nuestro trabajo, nos estaremos refiriendo a aquellos lectores de nivel inicial y de los primeros años (específicamente primer año de educación primaria).

De todos estos componentes, el que seleccionamos como mayormente vinculado a los procesos emocionales que el sujeto pone en juego a la hora de aprender a leer y escribir es el «comportamiento lector» (ProLEE, 2016), entendido como las formas de interacción y los tipos de práctica que el sujeto mantiene con la lectura en tanto se asume como lector. Algunos estudios lo han asociado con el componente afectivo de la actitud del sujeto hacia la práctica lectora (Stokman, 1999; Miesen, 2003; van Shooten, Globex y Stoel, 2004). Cabe destacar, en el aprendizaje inicial de la lectura, habilidades de orden emocional y afectivo facilitadoras del proceso, que se vinculan con aspectos motivacionales y actitudinales (Gallego, 2006). Por lo tanto, en el aprendizaje lector no 
solo intervienen aspectos de tipo cognitivo, sino también del desarrollo socioemocional (Castañeda, 1999).

En cuanto a la práctica de escritura, entendida como un sistema de signos articulados, arbitrarios y organizados según determinadas reglas y propiedades, como pueden ser la correspondencia grafema-fonema y la direccionalidad, entre otras, ProLEE la concibe (2016) como:

Una actividad inteligente, reguladora del pensamiento, que involucra las funciones psicológicas superiores, los diferentes niveles lingüísticos y el conocimiento del mundo del escritor. Implica un proceso consciente e intencional hacia el logro de objetivos previamente establecidos en el que convergen los mecanismos materiales requeridos para la ejecución, los procesos cognitivos implicados en la escritura y la situación social y cultural en la que estos acontecen (p. 57).

En la práctica de escritura, al igual que en referencia a la lectura, también se identifican componentes vinculados al sujeto que escribe, tales como comportamiento escritor, conocimiento del sistema de escritura, producción textual, conocimiento lingüístico y conocimiento discursivo. Así como en los componentes referidos a la práctica de lectura destacábamos el lugar del comportamiento lector, en la práctica de escritura destacamos el componente de comportamiento escritor. En este caso se hace referencia a los estilos o formas que el sujeto adopta para integrarse a una comunidad letrada, a las estrategias que pone en juego a la hora de escribir y que tienen relación con factores sensoriales, grafomotores, de atención y de memoria, y psicológicos o emocionales.

El siguiente cuadro resume, en las prácticas de lectura y escritura, las características que adopta el tipo de lector de nivel inicial y de los primeros años de educación primaria para el componente de comportamiento lector y escritor, según corresponda.

\section{Lenguaje y procesos psicológicos emocionales}

Partimos de concebir al lenguaje como instrumento de representación cognitiva, cuya adquisición supone la interacción social del sujeto con los demás (Pons et al., 2003). En este sentido, es importante comprender que, si bien la lectura y la escritura constituyen dos habilidades lingüísticas diferentes, ambas comparten los fundamentos del dominio de la habilidad comunicativa oral y metafonológica (Gutiérrez, 2014). Por lo tanto, se vuelve pertinente diferenciar el lenguaje hablado del lenguaje escrito. En el primer caso, nos referimos a la comunicación por medio de la lengua oral, la cual se apoya en dos funciones: la expositiva (como posibilidad de expresión) y la función de llamada (Buhler, 1934), como la posibilidad de actuar sobre el interlocutor. En tanto, el 
lenguaje escrito se diferencia del hablado fundamentalmente en tres aspectos: es más abstracto, más independiente de la situación y más consciente (Vygotski, 1979).

Tabla 1. Descriptores en lectura y escritura

\begin{tabular}{|c|c|c|}
\hline & Descriptores en lectura & Descriptores en escritura \\
\hline $\begin{array}{l}\text { Tipo de lec- } \\
\text { tor }\end{array}$ & $\begin{array}{l}\text { Componente comportamiento lec- } \\
\text { tor }\end{array}$ & $\begin{array}{l}\text { Componente comportamiento escri- } \\
\text { tor }\end{array}$ \\
\hline Lector inicial & $\begin{array}{l}\text { - Tiene interés por los libros. } \\
\text { - Sostiene la atención en instancias } \\
\text { de lectura colectiva brindadas por } \\
\text { el adulto. } \\
\text { - Manifiesta tener libros preferidos } \\
\text { y pide que se los lean. } \\
\text { - Hojea libros en forma individual y } \\
\text { grupal. } \\
\text { - Reconoce partes del libro. } \\
\text { - Identifica información paratex- } \\
\text { tual. } \\
\text { - Comienza a construir el concepto } \\
\text { de autoría. } \\
\text { - Memoriza a partir de la lectura } \\
\text { realizada por otros, por ejemplo: } \\
\text { una adivinanza o canción. }\end{array}$ & $\begin{array}{l}\text { - Manifiesta interés personal por } \\
\text { escribir. } \\
\text { - Utiliza soportes y herramientas } \\
\text { propios de la escritura. } \\
\text { - Comienza a reconocer la direccio- } \\
\text { nalidad de la escritura. } \\
\text { - Comienza a identificar algunos } \\
\text { usos sociales de la escritura, por } \\
\text { ejemplo: escribir para contar, para } \\
\text { solicitar algo, etc. } \\
\text { - Intenta apropiarse del código al- } \\
\text { fabético. } \\
\text { - Consulta a otros sobre la escri- } \\
\text { tura de palabras. } \\
\text { - Recurre y se apoya en diferentes } \\
\text { textos de su entorno para intentar } \\
\text { escribir. }\end{array}$ \\
\hline $\begin{array}{l}\text { Lector de } \\
\text { primer año }\end{array}$ & $\begin{array}{l}\text { - Recurre a la lectura por motiva- } \\
\text { ción personal, en soporte papel y } \\
\text { digital. } \\
\text { - Manifiesta preferencia por la lec- } \\
\text { tura de tipos de textos. } \\
\text { - Comienza a explorar textos con } \\
\text { independencia del lector experto. } \\
\text { - Utiliza distintas modalidades de } \\
\text { lectura pero con ayuda de un lector } \\
\text { experto. } \\
\text { - Lee con fines recreativos. }\end{array}$ & $\begin{array}{l}\text { - Empieza a apropiarse del sistema } \\
\text { de escritura. } \\
\text { - Se cansa ante la gran atención } \\
\text { que requiere la escritura. } \\
\text { - Manifiesta interés por registrar } \\
\text { diferentes eventos. } \\
\text { - Escribe con determinada fluidez. } \\
\text { - Recupera conocimientos previos } \\
\text { útiles. } \\
\text { - Asume el rol de autor de lo es- } \\
\text { crito. } \\
\text { - Atiende a la caligrafía. } \\
\text { - Se interesa por la escritura crea- } \\
\text { tiva. }\end{array}$ \\
\hline
\end{tabular}

Fuente: Adaptado de ProLEE, 2016. 
Cuanto mayores dificultades presenten los niños en la dimensión emocional, su representación simbólica de las emociones a través del lenguaje se verá también más limitada. Es decir que lenguaje y desarrollo de habilidades sociales y emocionales se influyen mutuamente. De acuerdo a las estadísticas, aproximadamente uno de cada cuatro niños que han sido diagnosticados con problemas emocionales presenta dificultades en el lenguaje y viceversa (Baixauli-Fortea, Roselló-Miranda y Colomer-Diago, 2015).

Las investigaciones realizadas en el campo de la salud mental infantil han demostrado que problemas de comportamiento internalizantes (miedo, inseguridad, apatía, tensión, ansiedad) y externalizantes (conductas disruptivas vinculadas a desobediencia, agitación psicomotora, agresividad) suelen ser los tipos de alteraciones psicológicas más comunes en niños a edades tempranas (Coronel, 2018). Así, cuando un niño pequeño se enfrenta al aprendizaje de la lectura y la escritura, pone en marcha diversos procesos psicológicos (Vallés, 2005), entre los cuales podemos distinguir procesos psicológicos básicos y procesos psicológicos de orden afectivo o emocional. En el primer caso nos referimos fundamentalmente a los tipos de atención y a la memoria (Vallés, 2005). En el segundo caso es posible deducir, de acuerdo a los aportes de Vallés (2005), los siguientes factores emocionales: estado de bienestar o de flujo (Csíkszentmihályi, 1998), manejo del estrés (Mayer y Salovey, 1997, Lazarus y Folkman, 1984), autoconcepto académico (González-Pienda y Núñez, 1998) y comportamiento motivacional (INEEd, 2017). A continuación desarrollaremos brevemente cada uno de estos procesos.

Estado de bienestar de flujo. Refiere a la experiencia de quien se enfrenta a la tarea de aprender siendo capaz de involucrarse activamente con la tarea, a la vez que disfrutarla. Esta teoría fue formulada en 1975 por el doctor en psicología Mihály Csíkszentmihályi (1998). Sus conclusiones derivan de haber investigado a cientos de expertos - artistas, músicos, médicos, atletas, académicos, entre otros - haciendo actividades que los apasionaban. A partir de los relatos de dicha investigación elaboró:

Una teoría de la experiencia óptima, basada en el concepto del flujo, el estado en el cual las personas se hallan tan involucradas en la actividad que nada más parece importarles; la experiencia, por sí misma, es tan placentera que las personas la realizarán incluso aunque tengan un gran coste, por el puro motivo de hacerla. (Csíkszentmihályi, 1998, p. 16)

El concepto expuesto nos lleva a pensar en la importancia de las condiciones en las que las tareas tienen lugar en el contexto educativo, donde en muchas ocasiones resultan poco placenteras o atractivas para los estudiantes. Esto, llevado al contexto de aprendizaje inicial de la lectura y la escritura, motiva a pensar en la necesidad de generar ambientes alfabetizadores y estimulantes para el desarrollo exitoso de experiencias. 
Manejo del estrés. Según el modelo de Mayer y Salovey (1997) sobre inteligencia emocional (IE), el manejo del estrés aparece como uno de los componentes principales de la IE. Para estos autores, la inteligencia, como habilidad mental específica, se relaciona con el uso adaptativo de las habilidades emocionales del sujeto. A partir de este componente se trabajan las llamadas estrategias de afrontamiento, que tienen que ver con la capacidad cognitiva que el sujeto despliega para afrontar las demandas internas (emocionales) o externas al realizar una actividad (Lazarus y Folkman, 1984). De esta manera, al momento de resolver determinada situación problema o en el cumplimiento de cierta tarea, el sujeto podrá orientar su acción a los factores externos o internos. Para este último caso, pueden aparecer conductas evitativas o de abandono de la actividad propuesta.

Autoconcepto. Cuando el niño forma parte de una situación de aprendizaje, intervienen en la experiencia diferentes ámbitos relacionados con su personalidad, que con el correr del tiempo logran estabilizarse y consolidarse como los estilos de aprendizaje propios del sujeto. Uno de estos ámbitos en los que el aprendizaje tiene lugar es el afectivo o emocional (González-Pienda, 1993; González-Pienda, Núñez y Valle, 1992), el cual se ve influido por factores tales como el autoconcepto. Es posible definir el autoconcepto como «un sistema complejo y dinámico de creencias que un individuo considera verdaderas respecto a sí mismo, teniendo cada creencia un valor correspondiente» (Purkey, 1970). En este sentido, investigaciones en torno a la temática de la influencia del autoconcepto sobre el rendimiento académico (González-Pienda, 1993; González-Pienda et al., 1992) han demostrado que es fuente de motivación respecto a los logros escolares.

Comportamiento motivacional. En el marco de las experiencias de aprendizaje, específicamente en el contexto de la enseñanza inicial de la lectura y la escritura, el comportamiento motivacional es un proceso psicológico integrado por metas, expectativas, atribuciones y otros componentes (INEEd, 2017). La motivación se concibe como el proceso a través del cual el sujeto tiene la capacidad de autorregular su actividad de aprendizaje. Esto refiere al monitoreo que hace de su proceso de cognición, a su motivación y su conducta bajo la influencia de los factores contextuales y ambientales.

Se suele hablar de cierta tendencia espontánea de los sujetos, desarrollada desde edades tempranas, que se vincula a un tipo de inclinación para el logro de determinado interés espontáneo. Este aspecto se conoce como motivación intrínseca. Si bien es concebido como una atribución innata, propia de los sujetos, existe evidencia de que todo proceso de motivación intrínseca requiere de puntos de apoyo (Reeve, 1996). En este sentido, las estrategias que los docentes favorecen a la hora de las experiencias de enseñanza y aprendizaje de la lectura y la escritura son relevantes como factores condicionantes para la facilitación u obstaculización de dicho proceso de motivación. 


\section{Programas de intervención en habilidades socioemocionales}

Una de las estrategias de acción y abordaje de las emociones en el ámbito escolar suelen ser los llamados proyectos o programas de intervención en educación emocional. Por programa de intervención entendemos un conjunto de actividades planificadas sistemáticamente, que se diseñan en torno a un marco conceptual y se proponen determinados objetivos, atendiendo a una aplicabilidad sostenida. Los programas suelen tener una organización que abarca los contenidos, las actividades, las estrategias de evaluación y un marco conceptual en el cual se fundamentan.

A continuación se describen algunos programas y proyectos de intervención en el área emocional, nacionales e internacionales, y se mencionan algunas de sus características. Como criterios de selección se adoptan los formulados por Fernández Sánchez y Jiménez-Dasí (2018): que la propuesta o parte de ella se enfoque en niños en edad preescolar; que tengan el propósito de abordar las habilidades socioemocionales; que se constituyan como propuestas secuenciadas, evaluadas y organizadas; que se dirijan a poblaciones universales, no en situaciones de riesgo ni con alteración del desarrollo. De acuerdo a ello, se describen las siguientes experiencias:

Programa Fundación Botín (España). Este programa tiene por objetivo favorecer el crecimiento físico, emocional, intelectual y social de las personas, promover la comunicación y la mejora en la convivencia de los centros escolares a partir del trabajo con docentes, alumnos y familias. Desde 2015, en coordinación con la ONG El Abrojo y la Universidad ORT Uruguay, el programa Educación Responsable comenzó a elaborar un piloto para nuestro país, derivado del programa de Fundación Botín, en cinco centros públicos de educación inicial, primaria y secundaria.

CASEL (Estados Unidos). Este proyecto tiene por objetivo promover el desarrollo de experiencias para la enseñanza de competencias académicas, sociales y emocionales. Además, busca contribuir con evidencia científica a la incorporación de la educación social y emocional en el sistema educativo, desde educación inicial hasta secundaria.

RULER. Se propone integrar al currículo escolar diferentes aspectos referidos a la inteligencia emocional. Ha logrado demostrar su incidencia en los logros académicos, la mejora en los niveles de agresividad de los alumnos y la reducción de la ansiedad.

Head Start, intervención REDI. Este programa de intervención temprana busca obtener impactos positivos sobre el lenguaje de los niños, las habilidades de alfabetización, la comprensión social y emocional, entre otros aspectos, llevados adelante con niños de 3 a 5 años pertenecientes a familias económicamente desfavorecidas.

Siento, luego aprendo (Gurises Unidos), Uruguay. Tiene por objetivo promover la incorporación de herramientas de trabajo que permitan a los maestros fortalecer el 
desarrollo emocional y facilitar el proceso de enseñanza y aprendizaje escolar de niños que asisten a escuelas ubicadas en barrios de nivel socioeconómico desfavorable en Uruguay.

SAT EDUCA Seekers After Truth (Buscadores de la verdad), Uruguay. Procura brindar a los docentes participantes herramientas para el trabajo personal y colectivo que fortalezcan especialmente dos de los tres ejes de formación permanente: la didáctica multigrado y el vínculo escuela-comunidad, a través de diversos módulos de trabajo. El primer módulo SAT para maestros rurales se realizó durante febrero de 2015 en el Centro Agustín Ferreiro, Canelones. Cuenta con el apoyo de la Fundación Claudio Naranjo.

Es importante pensar en los fundamentos de cada programa de intervención que se diseñe o aplique, sobre todo cuando la intervención se realiza con niños pequeños. $\mathrm{Al}$ respecto, Modzelewski (2016) propone no trabajar las emociones una por una, como es el enfoque de muchos programas, sino desde la autorreflexión, incluso en edades tempranas. Argumenta que aun los niños en edad preescolar, que no han logrado la autorreflexión, están en una etapa de desarrollo de esta facultad, por lo que las actividades de los programas deberían servir para estimular dicho proceso. Otro de los argumentos que expone es el referido a la relación entre lenguaje y emociones en los programas de intervención. Al respecto, establece:

El lenguaje, además, posibilita el desarrollo de la autorreflexión, que tiene lugar a través de este. Es por eso que, junto con el desarrollo de la autorreflexión, un programa de educación de las emociones debería prestar atención al uso del vocabulario emocional. ¿Cómo llamamos a determinada emoción que un compañero de clase está describiendo como propia, o a la emoción que está determinando las acciones de un personaje de un cuento? Este desarrollo va de la mano de la autorreflexión, son inseparables. (Modzelewski, 2016, p. 103)

Con estos fundamentos, sumados a la relación puesta en discusión sobre habilidades socioemocionales y aprendizaje de la lectura y la escritura, creemos necesario proponer programas de intervención que habiliten la estimulación de las habilidades socioemocionales durante la transición educativa de inicial a primer año. Estas propuestas de intervención han de partir de la importancia que el lenguaje tiene tanto para el desarrollo de la autorreflexión como del mecanismo para dar nombre a las emociones y así poder expresarlas. En este sentido, la literatura infantil puede ser un recurso relevante como eje mediador del trabajo con las habilidades socioemocionales en el ámbito escolar. 


\section{Conclusión}

Durante los primeros años de vida los niños terminan de desarrollar funciones primordiales que configurarán el lenguaje y las emociones, y más adelante lo harán con las competencias en lectura y escritura. Por ello, invertir en la primera infancia, evaluar tempranamente y detectar posibles dificultades es clave para el desarrollo infantil. En este trabajo se ha realizado un recorrido teórico que ha puesto en interacción las llamadas habilidades socioemocionales con el lenguaje, específicamente en las áreas de la lectura y escritura, guiado por dos interrogantes: ¿cómo influyen las habilidades socioemocionales en los desempeños esperados en lectura y escritura durante el primer año escolar? y ¿cómo promover una transición exitosa de inicial a primer año a través de experiencias de aprendizaje basadas en la estimulación de las habilidades socioemocionales? La premisa implicó considerar las habilidades socioemocionales como dinamizadoras de los desempeños cognitivos, en cuanto se presentan como factor modulador capaz de dificultar o facilitar el aprendizaje.

La emocionalidad ha estado presente a lo largo de la historiografía de lo escolar, pero en el último tiempo se advierte una especie de movimiento o giro educativo que pone las emociones en el centro. Concordamos en la relevancia del trabajo sobre las emociones, siempre que el propósito no esté puesto en su control o manejo como técnica de disciplinamiento, sino en la estimulación de las habilidades socioemocionales como favorecedoras de la práctica de autorreflexión y metacognición. Favorecer esto en las experiencias de enseñanza, aprendizaje y evaluación puede ser una oportunidad para estrechar las brechas de aprendizaje más allá de los factores ambientales o contextuales. Por otro lado, respecto al constructo de la emocionalidad, nos permitiría pensarlo desde lo epistémico y no como rutina educativa carente de argumentos psicopedagógicos y filosóficos.

El foco de este trabajo ha estado en la correlación del desarrollo emocional del sujeto, en cómo sus fortalezas y debilidades traducidas en habilidades socioemocionales influyen en su aprendizaje. Sobre esta premisa, la transición educativa de inicial a primer año merece ser abordada en su multidimensionalidad. Durante esta transición los actores involucrados se enfrentan a cambios muchas veces drásticos respecto a las dinámicas de trabajo, por lo que nos encontramos con prácticas que no siempre son pensadas desde la sensibilidad y la conciencia de la tensión emocional que puede operar en dicha transición.

Como estrategia de articulación educativa entre niveles, sería importante incorporar propuestas (programas) que articulen dinámicas de trabajo propias de la educación inicial que tengan continuidad durante el primer año escolar, de manera de favorecer las prácticas de enseñanza de lectura y escritura con un enfoque en que el compor- 
tamiento lector y escritor integre aspectos vinculados a las habilidades socioemocionales, como los procesos psicológicos (emocionales) que intervienen e interactúan con la dimensión cognitiva.

\section{Referencias}

Baixauli-Fortea, I., Roselló-Miranda, B., y Colomer-Diago, C. (2015). Relaciones entre trastornos del lenguaje y competencia socioemocional. Revista de Neurología, 60(S1), s51-s56.

Bisquerra, R. (2008). Educación para la ciudadanía: El enfoque de la educación emocional. Barcelona: Wolters Kluwer.

Bravo, V.L., y Salas, S. (1976). La repetición de curso en educación básica: Estudios del programa interdisciplinario de investigaciones en educación. Santiago de Chile: Universidad Católica de Chile.

Bülher, K. (1934). Theory of language: The representational function of language. Ámsterdam: John Benjamins.

Caballero García, A., y García-Lago Ibáñez, V. (2010). La lectura como factor determinante del desarrollo de la competencia emocional: Un estudio hecho con población universitaria. Revista de Investigación Educativa, 28(2), 345-359.

Castañeda, P.F. (1999). El lenguaje verbal del niño. Lima: Universidad Nacional Mayor de San Marcos.

Collaborative for Academic, Social, and Emotional Learning. (2013). Effective social and emotional learning programs: Preschool and elementary school edition. Chicago: CASEL.

Coronel, C.P. (2018). Problemas emocionales y de comportamiento en niños con discapacidad intelectual. Diversitas: Perspectivas en Psicología, 14(2), 351-362.

Cotrufo, T. (2016). En la mente del niño: El cerebro en sus primeros años. Barcelona: Salvat.

Csíkszentmihályi, M. (1998). Fluir: Una psicología de la felicidad. Barcelona: Kairós.

Durlak, J.A., y Weissberg, R.P. (2007). The impact of after-school programs that promote personal and social skills. Recuperado de https://casel.org/wp-content/uploads/2016/08/PDF-1-the-impact-of-after-school-programs-that-promote-personal-and-social-skills-executive-summary.pdf.

Durlak, J. A., y Weissberg, R. P. (2011). The impact of enhancing students' social and emotional learning: A meta-analysis of school-based universal interventions. Child Development, 82(1), 405-432.

Elias, M., Parker, S.J., Kash, M., Weissberg, R.P., y O’Brien, M.U. (2008). Social and emotional learning, moral education, and character education: A comparative analysis 
and a view toward convergence. En L. Nucci y D. Narvaez (eds.), Handbook of moral and character education (pp. 248-266). Nueva York: Routledge. Recuperado de https://www3.nd.edu/ dnarvaez/documents/Elias.pdf.

Elices, M. (2015). Emoción y cognición. En A. Vásquez Echeverría (ed.), Manual de introducción a la psicología cognitiva (pp. 223-248). Montevideo: Universidad de la República.

Escolano, A. (2018). Emociones y educación: La construcción histórica de la educación emocional. Madrid: Visión Libros.

Fernández-Berrocal, P., Pérez, J.C., Repetto, E. y Extremera, N. (2004). Una comparación empírica entre cinco medidas breves de inteligencia emocional percibida. En Proceedings of VII European Conference on Psychological Assessment (p. 61). Málaga: European Association of Psychological Assessment.

Fernández Sánchez, M., y Giménez-Dasí, M. (2018). Programas de intervención educativa para niños de 0 a 2 años. En M. Giménez Dasí y L. Quintanilla (coords.), Desarrollo emocional en los primeros años de vida: Debates actuales y retos futuros (pp. 197-210). Madrid: Pirámide.

Fondo de las Naciones Unidas para la Infancia (2012). Preparación para la escuela y las transiciones. Chicago: UNICEF.

Gallego, C. (2006). Los prerrequisitos lectores. Recuperado de http://alfabetizacionespanol.com/wp-content/uploads/2015/06/Predictores-lectores-2006-Gallego.pdf.

Gazmuri, V., Milicic, N., y Schmidt, S. (1978). Prevalencia de retardo mental y evolución de rendimiento escolar, en una muestra de 918 escolares del gran Santiago. Revista Chilena de Psicología, 1.

González-Pienda, J.A. (1993). Análisis del autoconcepto en alumnos de 6 a 18 años: Características estructurales, características evolutivo-diferenciales y su relación con el logro académico. Trabajo original de investigación. Oviedo: Universidad de Oviedo.

González-Pienda, J.A., y Núñez Pérez, J.C. (1998). Dificultades del aprendizaje escolar. Madrid: Pirámide.

González-Pienda, J.A., Núñez, J.C., y Valle, A. (1992). Influencia de los procesos de comparación interna/externa sobre la formación del autoconcepto y su relación con el rendimiento académico. Revista de Psicología General y Aplicada, 45(1), 73-82.

Gutiérrez, R. (2014). Interacción de los componentes del lenguaje oral en el proceso de aprendizaje de la lengua escrita. (Tesis doctoral). Universidad de Alicante, Alicante. Recuperado de https://rua.ua.es/dspace/bitstream/10045/39868/1/tesis_gutierrez_fresneda.pdf.

Instituto Nacional de Evaluación Educativa (2017). Informe de resultados de tercero y sexto de educación primaria. Montevideo: INEEd. 
Instituto Nacional de Evaluación Educativa (2018). Aristas: Marco de habilidades socioemocionales en sexto de educación primaria. Montevideo: INEEd.

James, W. (1945). Principios de psicología. Buenos Aires: Glem.

Kardowsky, W., Kovalsky, J., y Segure, T. (1973). Prevalencia de las alteraciones en el desarrollo intelectual de los escolares de 7 a 10 años en el área norte de Santiago. Trabajo presentado en el $14 .^{\circ}$ Congreso Panamericano del Niño, Chile.

Lazarus, R.S., y Folkman, S. (1984). Estrés y procesos cognitivos. Barcelona: Martínez Roca.

Marañón, G. (1985). Contribución al estudio de la acción emotiva de la adrenalina. Estudios de Psicología, 21, 75-89.

Mayer, J.D., y Salovey, P. (1997). What is emotional intelligence? En P. Salovey y D. Sluyter (eds.), Emotional development and emotional intelligence: Educational implications (pp. 3-31). Nueva York: Basic Books.

Miesen, H. (2003). Predicting and explaining literary reading: an application of the theory of planned behavior. Poetics, 31(3-4), 189-212.

Modzelewski, H. (2016). Fundamentos para un programa de educación de las emociones en una sociedad democrática. Revista de Investigación Social, 13(30), 83-110.

Montes Cruzat, M.; Condemarín, E., y Rioseco, R. (1984). Determinación del peso de la inmadurez en la repitencia y deserción en primer año básico de nivel socioeconómico bajo. Lectura y Vida, 5(3). Recuperado de http://www.lecturayvida.fahce.unlp.edu.ar/numeros/a5n3/05_03_Cruzat.pdf.

Organización para la Cooperación y el Desarrollo Económicos. (2019). PISA 2018: Assessment and analytical framework. doi:10.1787/b25efab8-en.

Pons, F., Lawson, J., Harris, P.L., y de Rosnay, M. (2003). Individual differences in children's emotions understanding: Effects of age and language. Scandinavian Journal of Psychology, 44, 347-353.

Programa de Lectura y Escritura en Español. (2016). Pautas de referencia sobre tipos

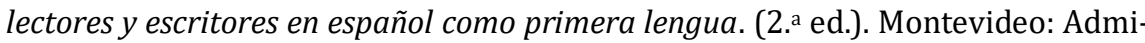
nistración Nacional de Educación Pública.

Purkey, W.W. (1970). Self-concept and school achievement. Englewood Cliffs, NJ: Prentice Hall.

Reeve, J. (1996). Motivación y emoción. Madrid: McGraw-Hill.

Salinas Quiroz, F. (2015). Educación inicial: Apego y desarrollo sociocognitivo. México: Universidad Pedagógica Nacional.

Schachter, S., y Singer, J. (1962). Cognitive, social, and physiological determinants of emotional state. Psychological Review, 69(5), 379-399.

Schiefelbein, E., y Farrel, J. (1973). Factores del proceso educativo chileno y sus efectos en el rendimiento de los alumnos. Santiago de Chile: Programa Regional Desarrollo Educativo. 
Stokmans, M. (1999). Reading attitude and its effect on leisure time reading. Poetics, 26(4), 245-261.

Terigi, F. (2009). Las trayectorias escolares: Del problema individual al desafío de política educativa. Buenos Aires: Ministerio de Educación.

Vallés, A. (2005). Comprensión lectora y procesos psicológicos. Liberabit, 11(11), 49-61. van Shooten, E., Glober, K., y Stoel, R. D. (2004). Development of attitude toward reading adolescent literature and literary reading behavior. Poetics, 32(5), 343-386.

Vásquez Echeverría, A., y Liz, M. (2019). El Inventario de Desarrollo Infantil (INDI): Características y avances del periodo 2015-2019. En S. Vernucci y E. Zamora (comps.), La ciencia de enseñar: Aportes desde la psicología cognitiva a la educación (pp. 44-52). Mar del Plata: Universidad Nacional de Mar del Plata.

Vygotski, L.S. (1979). Desarrollo de los procesos psicológicos superiores. Barcelona: Grijalbo.

Weissberg, R.P., Durlak, J.A., Domitrovich, C.E., y Gullotta, T.P. (2015). Social and emotional learning: Past, present, and future. En J.A. Durlak, C.E. Domitrovich, R.P. Weissberg, y T.P. Gullotta (eds.), Handbook of social and emotional learning: Research and practice (pp. 3-19). Nueva York: The Guilford Press. 